\title{
Rigorous derivation of the rate equations for the epitaxial growth
}

\author{
V I Tokar ${ }^{1,2}$ and H Dreyssé ${ }^{1}$ \\ ${ }^{1}$ IPCMS, UdS-CNRS, UMR 7504, 23 rue du Loess, F-67034 Strasbourg, France \\ ${ }^{2}$ Institute of Magnetism, National Academy of Sciences, 36-b Vernadsky Boulevard, \\ 03142 Kiev-142, Ukraine \\ E-mail: tokar@unistra.fr
}

\begin{abstract}
In the framework of the second-quantization representation of the master equation governing the irreversible epitaxial growth, exact equations describing the evolution of the island densities has been obtained. Their decoupling within a mean field-type approximation with the unknown correlation functions replaced by capture numbers $(\mathrm{CNs})$ has been used to derive a closed set of rate equations. The latter has been compared with the exact equations to obtain rigorous definitions of the CNs. The $\mathrm{CN}$ that describes the nucleation of dimer islands from two mobile monomers has been measured in the exact kinetic Monte Carlo simulations with the use of the rigorous definition. Strong disagreement with the literature values calculated within alternative techniques has been found, especially at low surface coverage. Plausible causes for the discrepancies are suggested.

Another important result of the rigorous approach is the rate equation with the term describing the monomer diffusion. The equation significantly differs from the widely used equations of this kind known from literature. Arguments in favour of our approach are given.

PACS numbers: $68.55 . \mathrm{A}-, 81.15 . \mathrm{Aa}$
\end{abstract}




\section{Introduction}

Lattice gas models (LGMs) with the gas atoms subject to stochastic kinetics are widely used in modelling the coherent growth on the surface [1, 2, 3]. The efficiency of the approach comes from two sources. First, the coherent LGM configuration can be fully characterized by the occupation numbers of a discrete lattice. This greatly reduces the phase space of the system which in more refined treatments is continuous and thus more difficult to deal with. Second, the stochastic kinetics of the LGMs allow for the use of the kinetic Monte Carlo (KMC) technique which in the case of discrete variables is capable of simulating the temporal evolution of the system on the experimental time scale in contrast to more accurate molecular dynamics methods which are restricted to very short times because of the computationally demanding continuous phase [2, 3].

In practical surface growth studies, however, even the use of the KMC meets with difficulties. For example, one of the most frequently studied quantities is the island size distribution [2] whose measurement requires gathering statistics on the number of islands of all sizes. Because in many cases the islands consist of several hundreds or even thousands of atoms [2], reliable statistics can be gathered only in repeated simulations of large systems. Therefore, sometimes additional arguments need be invoked to interpret the simulated data because of poor statistics [4]. Besides, there exist situations when the physics of the problem requires simulation of prohibitively large systems. For example, in the cases when the surface diffusivity is very large in comparison with the deposition rate, the KMC simulations of systems smaller then some critical value would lead to qualitatively incorrect results due to the finite size effects [5, 6]. Accelerated algorithms were developed to treat very large systems [7, 8] but their implementation in concrete cases meets with difficulties [9, 6].

Some of the difficulties with practical use of the KMC technique are a direct consequence of its strength, that is, of its ability to give a very detailed description of the system under study, such as, e. g., the precise morphologies of the growing islands. Such descriptions require considerable resources and efforts to obtain but are superfluous in the cases when only a coarse-grained picture of the growth is needed. For example, in [10] it was shown that to determine the parameters characterizing the atomic diffusivity on the surface in the sub-coalescence growth regime it is quite sufficient to gather experimental information only on the island densities with their morphologies being irrelevant.

Simplification of the modelling in such cases has been the subject of extensive studies (see, e. g., review articles [11, 2]). It was found that the island densities can be calculated with good accuracy in the framework of the mean field-type rate equations (REs) whose accurate solution requires only a fraction of the computational effort needed for the corresponding KMC study (see [12, 13, 5, 14, 2, 15, 16] and references therein). But this efficiency comes at the price of the necessity to independently assess many parameters or even functions entering the REs.

The number and the nature of the parameters depend on the specifics of the 
model under consideration. In the irreversible growth model that will be discussed in the present paper the input to the REs is a large (in theory infinite) set of the so-called capture numbers $(\mathrm{CNs})$ that describe the rates with which islands of various sizes incorporate diffusing atoms (or mobile monomers). The CNs has been the subject of extensive investigation since the introduction of the REs in this problem [13. In numerous studies a variety of functional forms and of the factors influencing the CNs were researched, such as the power-law dependence on the island size depending on the islands morphology [14], on the total island density (see [17, 18] and references therein), the influence of spatial correlations and capture zones, [19, 20, 21, 22, of selfconsistency conditions and total coverage [15, 23], etc.. Though considerable insights has been gained in these studies, fully satisfactory solution has not yet be found so new ideas are being put forward in in the search for accurate REs for the epitaxial growth [24, 25, 22].

One of the difficulties hampering the development of the theory is that in existing approaches the quality of the CNs is assessed either indirectly via the quality of the island size distributions they produce or with the use of an empirical procedure developed in [20, 2, 25]. A major goal of the present study is to propose a way to resolve this difficulty by suggesting a method of a direct measurement of the CNs in the KMC simulations. Our proposal is based on the observation that the stochastic KMC process can be exactly described by the corresponding master equation (ME) [26, 3]. With the use of the ME we can derive exact expressions describing the time evolution of the island densities. These expressions can be transformed into a closed set of REs by means of decoupling of a mean field kind. The CNs that appear in the decoupling can be compared to the exact expressions to obtain their precise meaning. With the use of these expressions the CNs will be "measured" in the exact KMC simulations. In the course of the derivation we will obtain an evolution equation containing the monomer diffusion term also found in the self-consistent approaches to the CNs calculation [15, 23, 27, 28]. Because our equation differs from the known ones and arguably is more accurate, we consider it as our second major achievement in this study. We hope that in the future this equation will allow us to improve the self-consistent theory of the CNs [15, 23, 27, 28].

The paper is organized as follows. In the next section we introduce the second quantization representation of the stochastic processes that describe the coherent epitaxial growth; in section 3 the exact evolution equations are derived and the REs obtained via their decoupling are presented in section 4, in particular, in section 4.1 we show how the conventional REs known from literature arise in our approach. In section 5 we will measure the capture rate for the nucleation of dimers in the so-called $\mathrm{i}=1$ model of irreversible growth [2]. Because the capture rate for the creation of dimers effectively defines the total island density [15], its accurate definition may improve the estimates of the surface diffusion on the basis of experimental data [10]. In the concluding section 6] we briefly discuss the results obtained and possible farther development. 


\section{Master equation in the second quantization representation}

The quantum formalism in application to stochastic LGMs was introduced in [29, 30, 31 ] and subsequently applied by many authors to various stochastic models (for further bibliography see [32, 33]). The formalism does not introduce any new physics into the problem but allows one to efficiently deal with huge matrices that appear in the ME approach in the kinetic many-body problems using the powerful tools from the second quantization or the quantum field theories.

For concreteness we will illustrate the formalism using the simple model of submonolayer growth previously studied in, e. g., [25]. In this model the substrate has the square lattice geometry. It is assumed that each site can be occupied by one atom at most. Thus, the system consisting of $M$ sites the number of configurations is $2^{M}$.

\subsection{The Fock representation of the state space}

Usually in LGMs the atomic configuration is characterized by the set of the occupation numbers

$$
\left\{n_{i}=0,1\right\},
$$

where $i$ is the lattice site index. But it is obvious that any quantity acquiring two values can be used. In the quantum formalism the state of the system under consideration which we denote by the "ket" vector $|\alpha\rangle$ can be characterized by the product over all lattice sites

$$
|\alpha\rangle=\prod_{i}\left|b_{i}\right\rangle_{i}, \quad b_{i}=0,1
$$

of the binary vectors

$$
|0\rangle_{i}=\left(\begin{array}{l}
0 \\
1
\end{array}\right)_{i} \text { and }|1\rangle_{i}=\left(\begin{array}{l}
1 \\
0
\end{array}\right)_{i},
$$

where the first vector corresponds to the empty site and the second vector to the occupied one. Now, by introducing corresponding conjugate "bra"-vectors via the transposition of vectors (3) one can see that the set of state vectors (2), besides being complete, is also orthonormal.

Transitions between the states of different occupation in (3) are performed with the use of the creation and annihilation operators of the hard-core bosons [31, 33]

$$
a_{i}^{+}=\left(\begin{array}{cc}
0 & 1 \\
0 & 0
\end{array}\right)_{i} \text { and } a_{i}=\left(\begin{array}{cc}
0 & 0 \\
1 & 0
\end{array}\right)_{i}
$$

In the space of vectors (2) of dimensionality $2^{M}$ the matrices have the size $2^{M} \times 2^{M}$. Therefore, the action of operators (44) should be augmented with unit $2 \times 2$ matrices acting on the sites different from $i$. For simplicity in derivations below we omit these trivial factors. 
As is easily verified with the use of the explicit representations above, $a_{i}$ and $a_{i}^{+}$ satisfy all the usual properties of the creation and annihilation operators

$$
a_{i}^{+}|0\rangle_{i}=|1\rangle_{i}, \quad a_{i}|1\rangle_{i}=|0\rangle_{i} .
$$

In particular, the operator whose eigenvalues correspond to the occupation numbers (1) has the conventional form (for simplicity here and below we will use the same notation for the operator and for the occupation numbers)

$$
n_{i}=a_{i}^{+} a_{i}=\left(\begin{array}{ll}
1 & 0 \\
0 & 0
\end{array}\right)_{i}
$$

as is easily seen from (4). Despite the no-double-occupancy conditions that follow from the definitions (4) and are reminiscent of the fermions:

$$
\left\{a_{i}^{+}, a_{i}\right\}=1 \text { and }\left(a_{i}^{+}\right)^{2}=a_{i}^{2}=0
$$

(the braces denote the anticommutator), the operators at different sites commute:

$$
\left[a_{i}^{+}, a_{j}\right]_{i \neq j}=0 \text {. }
$$

Finally, by defining the vacuum state of the system as that of the empty lattice

$$
|0\rangle=\prod_{i}|0\rangle_{i}
$$

we can represent the state vectors (2) of the system as the Fock space

$$
|\alpha\rangle=\left(\prod_{\text {Occupied sites }} a_{i}^{+}\right)|0\rangle .
$$

\subsection{Master equation in the Fock space}

The stochastic LGM of the surface growth that we consider in the present paper belongs to the general category of stochastic models whose kinetics satisfy the ME

$$
\frac{d P_{\alpha}}{d t}=\sum_{\beta}\left(W_{\alpha \beta} P_{\beta}-W_{\beta \alpha} P_{\alpha}\right),
$$

where $P_{\alpha}(t)$ is the probability of the system to be found at time $t$ in state $\alpha$ and $W_{\alpha \beta}$ is the matrix of transition rates between different states of the system. As was shown above, in the case of the growth on the surface in the representation of occupation vectors (3) the matrix has $M$ binary indices and its size is $2^{M} \times 2^{M}$. Dealing with such matrices in the case of macroscopic surfaces $\left[M \sim O\left(10^{15}\right)\right]$ is not an easy task. But as we saw on the example of the creation and annihilation operators (4), the structure of the $2^{M} \times 2^{M}$ matrix can be rather trivial consisting mostly from the Kronecker product of the $2 \times 2$ identity matrices. The Fock space formalism emphasizes the nontrivial aspects of the transition matrix leaving trivialities behind the scene. For example, a simple model of the surface growth consisting only in atomic deposition can be described within this formalism by the operator

$$
\mathbf{W}^{F}=F \sum_{i} a_{i}^{+}
$$


where $F$ is the deposition rate. Using explicit representation for the state vectors (2) and the operator identities (7) it is easy to see that the matrix element

$$
W_{\alpha \beta}^{F} \equiv\left\langle\alpha\left|\mathbf{W}^{F}\right| \beta\right\rangle=\left\{\begin{array}{l}
0 \\
F
\end{array}\right.
$$

is different from zero only provided $|\alpha\rangle=a_{i}^{+}|\beta\rangle$ for some site index $i$ and the site is empty in state $|\beta\rangle$ because according to (7) two atoms cannot sit on the same site. Thus, the transition matrix in this case is very sparse despite being very large. Similarly, hopping diffusion of a free atom on the surface can be described with the use of the operator

$$
\mathbf{W}_{0}^{D}=D \sum_{i i_{\mathrm{N}}} a_{i_{\mathrm{N}}}^{+} a_{i},
$$

where $D$ is the diffusion rate and we assumed that the atom that left site $i$ may hop only to one of four nearest sites denoted here and in the following by the subscript $\mathrm{N}$.

Further, using the complete set of the system states, it is convenient to cast the ME (11) into the form

$$
\frac{d|t\rangle}{d t}=\mathbf{T}|t\rangle,
$$

where

$$
|t\rangle=\sum_{\alpha} P_{\alpha}(t)|\alpha\rangle
$$

and

$$
\mathbf{T}=\left[W_{\alpha \beta}-\delta_{\alpha \beta} \sum_{\gamma} W_{\gamma \alpha}\right] .
$$

In practice one is usually interested in the average values of various densities described by the operators that are diagonal in the Fock space and can be calculated as

$$
O(t)=\langle\hat{O}\rangle(t)=\sum_{\alpha} P_{\alpha}(t)\langle\alpha|\hat{O}| \alpha\rangle .
$$

To express such averages in the form of matrix elements, as is conventional in quantum formalism, we introduce the matrix containing all possible states of the system with equal (unit) weight as

$$
|\Omega\rangle=\sum_{\beta}|\beta\rangle .
$$

Now using the orthonormality of the state vectors one gets

$$
O(t)=\langle\Omega|\hat{O}| t\rangle .
$$

Another important observation concerning vector $|\Omega\rangle$ in (19) is that, as can be seen from (17), the sum of the matrix elements of matrix $\mathbf{T}$ over the left index is equal to zero: $\sum_{\alpha} T_{\alpha \beta}=0$. This is the consequence of the fundamental requirement of the probability conservation and in the quantum notation can be written as

$$
\langle\Omega| \mathbf{T}=0 .
$$


Now using this identity and formally solving (15) via the operator exponent and assuming that the initial state at $t=0$ is the vacuum state (9) (the empty lattice) one can re-write (20) as

$$
O(t)=\left\langle\Omega\left|e^{-t \mathbf{T}} \hat{O} e^{t \mathbf{T}}\right| 0\right\rangle .
$$

From this we finally obtain an exact equation for the time evolution of the average value of $O(t)$ :

$$
\frac{d O}{d t}=\langle\Omega|[\hat{O}, \mathbf{T}]| t\rangle
$$

\section{Evolution of island densities during submonolayer growth}

To derive the evolution equations for the island densities one needs to know the operators $\mathbf{T}$ and $\hat{O}$ entering (23). We first note that it suffices to know only the first non-diagonal term of $\mathbf{T}$ in (17) because the density operators we are interested in are diagonal and thus will commute with the diagonal part in (23). (In case of necessity the diagonal part of $\mathbf{T}$ can be easily recovered from the non-diagonal part given below [33].) The operator W that describes the epitaxial growth in the model under consideration consists of only two terms: the deposition term (12) and the diffusion term of the type of (14) modified to include interatomic interactions through the dependence of the diffusion rate on the atom environment:

$$
\mathbf{W}=F \sum_{i} a_{i}^{+}+\sum_{i, i_{\mathrm{N}}} a_{i_{\mathrm{N}}}^{+} D_{i} a_{i} .
$$

This is done with the use of the activated dynamics that is conventionally used in the surface growth studies [34, 15, 11]:

$$
\begin{aligned}
& D_{i}=D \exp \left[-\left(E_{N} / k T\right) \sum_{i_{\mathrm{N}}} n_{i_{\mathrm{N}}}\right]=D \prod_{i_{\mathrm{N}}} \exp \left[-\left(E_{N} / k T\right) n_{i_{\mathrm{N}}}\right] \\
& =D \prod_{i_{\mathrm{N}}}\left(1+\left[\exp \left(-E_{N} / k T\right)-1\right] n_{i_{\mathrm{N}}}\right) \stackrel{E_{N} \gg k T}{\longrightarrow} D \prod_{i_{\mathrm{N}}} \bar{n}_{i_{\mathrm{N}}} .
\end{aligned}
$$

Here $E_{N}>0$ is the energy of binding between neighbour atoms, in the second row use has been made of the property $n_{i}^{2}=n_{i}$, and $\bar{n}_{i}=1-n_{i}$. The irreversible growth corresponds to the limit shown at the end of (25) so in the calculations below we will use the diffusion term in the form

$$
\mathbf{W}^{D}=D \sum_{i i_{\mathrm{N}}} a_{i_{\mathrm{N}}}^{+} \tilde{a}_{i},
$$

where according to (25)

$$
\tilde{a}=a_{i} \prod_{i_{\mathrm{N}}} \bar{n}_{i_{\mathrm{N}}}
$$

Next we need to define the island density operators. We define atomic islands on the square lattice similar to the definition of the lattice animals [35], namely, as the clusters of atoms occupying the sites any pair of which can be connected by a path that 


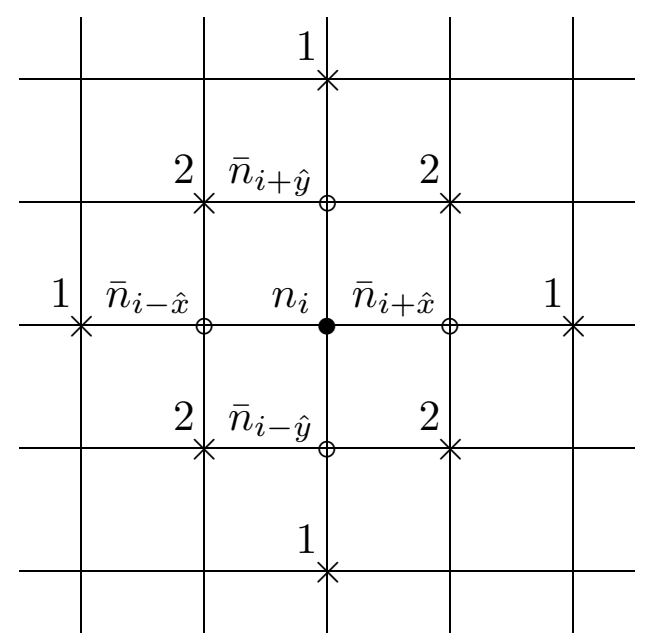

Figure 1. Encircled sites illustrate the structure of the monomer operator $\hat{N}_{1, i}$ in (29). The crossed sites are the next neighbours (NN) to site $i$ and the numbers are the values of $m_{i_{\mathrm{NN}}}$ (the numbers of nucleation paths) in (33).

traverses only nearest neighbour sites all belonging to the cluster. We characterize the island by its size $s$, configuration $c$, and the position on the lattice $i$. The latter can be defined arbitrarily but uniquely for all island of given configuration. The islands have different configurations if they cannot be obtained from each other by lattice translations. According to this definition, the dimers oriented along axis $x$ and along axis $y$ have different configurations. Thus, the density operator for the cluster of any size (including both the islands and the monomers) that has configuration $c$ and is placed at cite $i$ is

$$
\hat{N}_{s, i}^{(c)}=\prod_{\{j \in c\}} n_{j} \prod_{\left\{j_{\mathrm{N}} \in \bar{c}\right\}} \bar{n}_{j_{\mathrm{N}}} .
$$

The second product in this expression is over the sites bordering the island by which we mean all sites that do not belong to the island but are nearest neighbour to at least one island site (we will denote the set of such sites as $\bar{c}$ ). Operators $\bar{n}_{j_{N}}$ are needed to guarantee that the island contains exactly $s$ atoms: without this factor the operator $\hat{N}_{s, i}^{(c)}$ could produce nonzero expectation value at configurations $c$ that make parts of larger islands. In the case of monomer operator $\hat{N}_{1, i}$ the superscript $c$ is not needed because the monomer configuration is unique (see figure 1):

$$
\hat{N}_{1, i}=n_{i} \prod_{\hat{e}= \pm \hat{x}, \pm \hat{y}} \bar{n}_{i+\hat{e}}
$$

where $\hat{x}$ and $\hat{y}$ are the unit lattice vectors.

\subsection{Evolution equations for the island densities}

Substituting the island density operators (28) into (23) and calculating the commutator (see 6) one arrives on the evolution equations for their average values. The densities are 
calculated according to the usual expressions:

$$
\begin{aligned}
& N_{s}^{(c)}=M^{-1} \sum_{i} N_{s, i}^{(c)}, \\
& \left\langle\hat{N}_{1, i_{\mathrm{NN}}} \hat{N}_{s}^{(c)}\right\rangle=M^{-1} \sum_{i}\left\langle\hat{N}_{1, i_{\mathrm{NN}}} \hat{N}_{s, i}^{(c)}\right\rangle, \text { etc. }
\end{aligned}
$$

In the first equation the carets over the symbols are omitted because here they are not operators but the number densities calculated according to (20); in the second equation and in the following the subscripts $i_{\mathrm{N}}$ or $i_{\mathrm{NN}}$ are considered to be relative coordinates with respect to the corresponding cluster or site (nearest- or next-nearest neighbours) and thus in (31) they are not averaged out.

Thus, for the islands $(s>1)(23)$ with the use of (A.11) and (A.10) takes the form $\frac{d N_{s}^{(c)}}{d t}=D \sum_{\{c \backslash 1\} i_{\mathrm{NN}}}^{\prime}\left\langle\hat{N}_{1, i_{\mathrm{NN}}} \hat{N}_{s-1}^{(c \backslash 1)}\right\rangle-D \sum_{i_{\mathrm{NN}}} m_{i_{\mathrm{NN}}}\left\langle\hat{N}_{1, i_{\mathrm{NN}}} \hat{N}_{s}^{(c)}\right\rangle+F \sum_{\{c \backslash 1\}} N_{s-1}^{(c \backslash 1)}-F \bar{s}_{c} N_{s}^{(c)}+\ldots$

The prime over the first sum reminds that the summation over $i_{\mathrm{NN}}$ is not over all NN sites to the $s-1$-atom island but only over the NN positions that the detached atom can reach and remain free. By the dots we denoted the terms responsible for the coalescence. It would not be too difficult to write down these terms. Because of the symmetry of the square substrate a diffusing atom can be caught by one, two or three nearby clusters (islands and/or monomers). Similarly, the process of direct impingement can include up to four clusters. Thus, as can be seen, these processes could have been accounted for in (32) with the use of 19 additional terms containing all admissible combinations of islands and monomers. Inclusion of these terms, however, would have made the equation very cumbersome. Besides, currently the research activity in the field of the irreversible growth is centred around the pre-coalescence regime where only the processes taken into account in (32) participate. This regime corresponds to smaller coverages $\theta \lesssim 0.2$ and physically describes the ensemble of well separated isolated islands interspersed with low density monomer gas. Because the density of all clusters are very low the processes between closely spaced three or four clusters are small and can be neglected in this regime. If, however, there is need to account for such processes (at high coverages, for example), our technique allows for rigorous derivation of necessary equations. But in the present paper we will neglect the coalescence terms in all equations below.

In order to include spatial correlations and/or fluctuations into the mean-field description, equation describing the monomer diffusion have been proposed [15, 23, 28, 36]. To obtain similar equation in our approach we postpone the site averaging in (30) corresponding to $\hat{N}_{1, i}$ till later. Now from (A.14) and (A.12) one gets

$\frac{d N_{1, i}}{d t}=F N_{0, i}-4 F N_{1, i}+D\left(\sum_{i_{\mathrm{N}}}^{\prime} N_{1, i_{\mathrm{N}}}-4 N_{1, i}\right)-\sum_{i_{\mathrm{NN}}} m_{i_{\mathrm{NN}}}\left\langle\hat{N}_{1, i_{\mathrm{NN}}} \hat{N}_{1, i}\right\rangle$

where $N_{0, i}$ is defined in (A.13) and the prime on the summation over $i_{\mathrm{N}}$ means that the corresponding terms are present only if site $i$ does not have nearest neighbour atoms. This is a consequence of the irreversible dynamics we are studying. If the atom at $i$ had 
a neighbour it would have been bound and so could not produce a free monomer via the diffusion hop.

The comparison of (33) with the equations proposed in [15, 28] as well as derivation with its help of the conventional site averaged rate equation for $N_{1}$ will be given in the next section.

\section{Rate equations}

The equations (32) and (33) cannot be used directly for the calculation of island densities because they contain unknown terms - the averages of the operator products in the angular brackets. The standard way to proceed in such cases is to decouple them by replacing the mean of the operator product with the product of the means

$$
\left\langle\hat{N}_{1, i_{\mathrm{NN}}} \hat{N}_{s}^{(c)}\right\rangle \approx N_{1} N_{s}^{(c)} .
$$

This is the conventional mean field approximation that proved its efficiency in a lot of many-body problems. In the theory of epitaxial growth, however, the standard meanfield approach was found to be too crude [2]. Therefore, it was suggested to account for the correlations neglected in (34) via the use of parameters $\sigma_{s}^{(c)}$ defined according to the equality

$$
\sum_{i_{\mathrm{NN}}}\left\langle\hat{N}_{1, i_{\mathrm{NN}}} \hat{N}_{s}^{(c)}\right\rangle=\sigma_{s}^{(c)} N_{1} N_{s}^{(c)} .
$$

Because of their physical meaning the parameters are called the capture numbers [2]. Substituting (35) into (32) and augmenting the set by the equation for the monomer density derived in 4.1 we arrive at a formally closed set of equations that describe the evolution of the island densities. The set, however, is not complete without explicit expressions for the capture numbers. As we noted in the Introduction, there exist many heuristic approaches aiming to define the CNs independently. Our aim in the present paper is to propose a method to derive the exact values of the CNs from the KMC simulation data. This would provide us with a definite mean of assessing the quality of the CNs obtained in empirical approaches. We achieve this by noting that equations (32) and (33) are rigorously derived from the ME (11) that exactly describes the KMC stochastic process. Thus, the KMC data should satisfy these equations exactly hence by comparing them with the empirical REs one can calculate the exact values of CNs. For example, (35) can be trivially solved as

$$
\sigma_{s}^{(c)}=\sum_{i_{\mathrm{NN}}}\left\langle\hat{N}_{1, i_{\mathrm{NN}}} \hat{N}_{s}^{(c)}\right\rangle / N_{1} N_{s}^{(c)} .
$$

We note that all quantities on the right hand side of this expression can be measured in the course of exact KMC simulations thus providing the exact values of CNs. A comment is in order concerning the influence on this result of the coalescence terms that we omitted in our evolution equations. It should be noted that they also are discarded in the REs that we are studying in this paper [5, 2] and that the CNs cannot be calculated with better accuracy than their definition allows. Thus, for the equations 
derived in the "no coalescence" approximation in the KMC simulations we may count only those configurations where exactly one monomer and one island participate and discard those processes which lead to coalescence. In this sense the measurements of CNs with the use of (36) and KMC may be considered to be in exact correspondence with its definition.

Individual capture numbers for every island configuration introduced in (35) and (36) can be efficient in the cases when the configurations are easily enumerable, e. g., in the case of one-dimensional islands either on the one-dimensional (1D) substrate or on 2D substrates in the cases of very anisotropic quasi-1D growth [37, 38, 4]. Because 1D islands have only one configuration characterized by their size $s$, the superscript $(c)$ for such islands in the above equations is superfluous.

In more common case of isotropic growth in $2 \mathrm{D}$, however, the number of island configurations is so large that for $s \gtrsim 50$ even their enumeration is infeasible [35]. In such cases cruder description of island morphologies is needed. Usually only the island size statistic is being gathered both in the KMC simulations and in the growth experiments. So now we derive the formulas generalizing (36) to this case.

\subsection{REs in isotropic case}

Because the number of configurations of islands of sizes $s \gtrsim 50$ is unmanageable [35], the rate equations are usually written for the total density of islands containing the same number of atoms as

$$
N_{s}=\sum_{c} N_{s}^{(c)}
$$

The standard REs for the island densities during the irreversible growth have the form [2]

$\frac{d N_{s}}{d t}=D N_{1}\left(\sigma_{s-1} N_{s-1}-\sigma_{s} N_{s}\right)+F \kappa_{s-1} N_{s-1}-F \kappa_{s} N_{s}, \quad s=2,3, \ldots$

We can derive them from (32) by summing the latter over the configurations corresponding to the same island size as in (37). In this way we obtain the left hand side of (38). But on the right hand side one finds the terms of the kind

$$
-F \sum_{c} \bar{s}_{c} N_{s}^{(c)}
$$

[see the last term in (32)] Identifying it with the last term in (38) we arrive at the following definition of the capture numbers for direct impingement

$$
\kappa_{s}=\sum_{c} \bar{s}_{c} N_{s}^{(c)} / N_{s}
$$

One may wonder whether this definition is consistent with the positive deposition terms in (32) and (38) because the positive term in (32) has the form quite different from that of the negative term. To see that they acquire the same form after the summation over configurations it is sufficient to note that every term of the kind $N_{s-1}^{(c \backslash 1)}$ will enter the sum proportional to $F$ in (32) as many times as there is configurations with $s$ atoms 
that would lead to the configuration $(c \backslash 1)$ when the atom is taken away. Obviously that the atoms can be taken only from one of the sites in the configuration border so there is exactly $\overline{(s-1)}_{(c \backslash 1)}$ such terms.

Similarly, using the second term on the first line of (32) and comparing it with (38) one gets

$$
\sigma_{s}=\sum_{i_{\mathrm{NN}, \mathrm{c}}} m_{i_{\mathrm{NN}}}\left\langle\hat{N}_{1, i_{\mathrm{NN}}} \hat{N}_{s}^{(c)} / N_{1} N_{s}\right.
$$

The contribution of the first (positive) term in (32) can be analysed similar to the direct impingement case discussed above.

To close the set of the rate equations for the island densities, the equation for $N_{1}=M^{-1} \sum_{i} N_{1, i}$ is needed. Its left hand side is trivially obtained by averaging the left hand side of (33) over the surface, so let us consider in detail the right hand side of this equation. The first term the "empty island" operator gives a nonzero contribution equal to unity only when site $i$ and all its neighbours are empty. This excludes all occupied sites as well as the sites bordering the islands and the monomers. Taking into account (40) and the second term in (33) which is simply an additional monomer contribution $-\kappa_{1} N_{1}\left(\kappa_{1}=4\right)$ we obtain the term in the parentheses of the conventional RE for the monomers (see, e. g., [25]):

$\frac{d N_{1}}{d t}=F\left(1-\theta-2 \kappa_{1} N_{1}-\sum_{s>1} \kappa_{s} N_{s}\right)-2 D \sigma_{1} N_{1}^{2}-D N_{1} \sum_{s>1} \sigma_{s} N_{s}$.

The diffusion term in (33) is the discrete Laplacian that consists of two terms: the positive contribution from the monomer hops on site $i$ from four neighbour sites with monomer densities $N_{1, i_{\mathrm{N}}}$ and the negative contribution from site $i$ on the neighbour sites. Thus, when summed over the whole lattice the negative terms tend to be cancelled by the positive contributions from the neighbour sites. In the presence of islands and other monomers, however, the positive terms may not exist because of the occupied sites nearest to them. This leaves uncompensated terms in the negative contribution that equals exactly to the number $m_{i_{\mathrm{NN}}}$ of the nucleation paths to the mentioned islands or monomers [23]. This in accordance with (41) gives the last two negative terms in (42). Finally, the contribution that gives factor 2 to $\sigma_{1}$ term comes from the last term of (33).

\subsection{Comparison with equations from the self-consistent approach to the CNs calculation [15]}

To facilitate the comparison we first rewrite (33) in the continuous substrate coordinates $i \rightarrow \mathbf{x}$ as

$$
\frac{d N_{1}(\mathbf{x})}{d t} \simeq F N_{0}(\mathbf{x})-\kappa_{1} F N_{1}(\mathbf{x})+D \nabla^{2} N_{1}(\mathbf{x})-D \sigma_{1} N_{1}(\mathbf{x})^{2},
$$

where $N_{0}(\mathbf{x})$ is the continuous version of the "empty island" operator $\hat{N}_{0, i}$ from (33). 
In this notation equation (11) from [15] would read (we rearranged some terms for convenience)

$$
\begin{aligned}
\frac{d N_{1}(\mathbf{x})}{d t} \simeq & F-\kappa_{1} F N_{1}(\mathbf{x})+D \nabla^{2} N_{1}(\mathbf{x})-2 D \sigma_{1} N_{1} N_{1}(\mathbf{x}) \\
& -F \sum_{s=1}^{\infty} \kappa_{s} N_{s}-D N_{1}(\mathbf{x}) \sum_{s>1}^{\infty} \sigma_{s} N_{s}
\end{aligned}
$$

where

$$
N_{1} \equiv \frac{1}{M a^{2}} \int d \mathbf{x} N_{1}(\mathbf{x}) \simeq \frac{1}{M} \sum_{i} N_{1, i}
$$

(the lattice constant $a$ is assumed to be equal to unity). In comparing (44) with (43) we first note that the last term in (43) that describes the island nucleation is local in $\mathbf{x}$. This is reasonable because the adatoms nucleate an island when meeting at one spatial point in the continuum approximation (or at the nearest sites on the lattice) while according to (44) and (45) the adatom at point $\mathbf{x}$ nucleates an island with any other adatom in the system which is unphysical. Secondly, in (44) the nucleation term enters with the coefficient 2 , in contrast to our equation (43). The origin of this discrepancy is connected with the last term of (44) that is absent in our equation (43) but is present as the last term in (42)). As we saw in section 4.1, it arises from the averaging the discrete Laplacian in (33) over the whole surface. Thus, in the continuum approximation this term also should appear from the integration of $\nabla^{2} N_{1}(\mathbf{x}) \equiv \operatorname{div}\left[\nabla N_{1}(\mathbf{x})\right]$ as the sink term for the monomer attachment to the island boundaries. But as it is already present in (44) this means that the capture of monomers is counted twice in this equation, unless some very special treatment of the boundary conditions is undertaken which seems to be the case in 15 .

Similarly, the first term on the second line in (44) appears in our formalism only after the first term in (43) is averaged over the whole surface, as in (42)). These direct impingement terms are less important under the usual growth conditions and are often neglected, though they may became important in some cases. Apart from these terms, our equation (43) is very similar to the $i=1$ case of the equation derived in [27, 28]. In this approach the nucleation is local and the capture term $D N_{1}(\mathbf{x}) \sum_{s>1}^{\infty} \sigma_{s} N_{s}$ is absent from equation (2.7) in [28]. The nucleation term, however, enters with the coefficient $i+1=2$ which we consider to be double counting. Careful comparison of all three equations with exact KMC simulations is necessary to clarify this complicated issue.

\section{The nucleation rate}

To illustrate the above formalism, in this section we present the calculation of $\sigma_{1}$ with the use of the KMC simulations and the formula

$$
\sigma_{1}=\sum_{i_{\mathrm{NN}} \in \mathrm{NN}} m_{i_{\mathrm{NN}}}\left\langle\hat{N}_{1, i_{\mathrm{NN}}} \hat{N}_{1, i} /\left(M N_{1}^{2}\right)\right.
$$


that can be derived from the last term in (33), as explained in 4.1. As was noted in the Introduction, $\sigma_{1}$ is the most important of the $\mathrm{CNs}$ in the systems belonging to the so-called $\mathrm{i}=1$ case when all islands with $s>1$ are stable. This is because in the usually considered case of large $R$ when the direct impingement terms are small, $\sigma_{1}$ effectively defines the nucleation of dimers and hence the total number of islands in the system because every island nucleates as a dimer in the $i=1$ case [15]. The total island density measured experimentally can be used to define the diffusivity of atomic monomers on the surface [10]. Therefore, an accurate theoretical calculation of $\sigma_{1}$ could lead to more reliable empirical estimates of the microscopic parameters that define the surface diffusivity. Because of this, $\sigma_{1}$ was calculated by several authors but controversies remained. For example, the widely cited analytic expression [39, 5, 15, 2]

$$
\sigma_{1} \simeq \frac{4 \pi}{\ln \left(C R N_{1}\right)}
$$

where $C$ is a numerical constant. In the steady-state regime at large $R$ the monomer density behaves as [5]

$$
N_{1} \sim\left(3 \pi^{2} \theta R^{2}\right)^{-1 / 3} .
$$

It should be noted that this formula is non-physical at small coverage where $N_{1} \approx \theta$ while according to (48) $N_{1}$ diverges. Therefore, we will use it only above the crossover coverage separating the transient and the steady-state regimes [2]

$$
\theta^{*}=R^{-1 / 2}
$$

(see figure 21). Thus, substituting (48) into (47) one gets

$$
\sigma_{1} \simeq \frac{4 \pi}{\ln C_{1}+(\ln R-\ln \theta) / 3},
$$

where $C_{1}=C /\left(3 \pi^{2}\right)^{1 / 3}$. As is seen, the dependence on $R$ in (50) is formally as strong as on $\theta$ and in practice is even stronger because in experiments and simulations on growth in precoalescence regime the coverage usually varies within one order of magnitude $(\theta \sim 1-20 \%)$ while $R$ varies over several orders of magnitude. For example, in simulations of [25]) $R=10^{5}-10^{8}$. Yet the authors arrive at the conclusion that the coverage dependence is more important.

To compare the values of $\sigma_{1}$ calculated in the framework of the above theories with the exact values given by (46) we performed KMC simulations at two values of $R$ and at small coverage shown in figures 2 and 3 . The choice of parameters was dictated by the necessity to obtain reasonable statistics which depend on the monomer density $N_{1}$, as can be seen from (46). The latter diminishes with the growth of both $R$ and $\theta$, as is seen from (48). Therefore, we have chosen the region near the crossover coverage (49) where the density is at its maximum (see figure 2) [2].

In the harder $R=10^{5}$ case, $400 \mathrm{KMC}$ runs in the system with $4096 \times 4096$ sites were made. In the easier $R=10^{4}$ case $2048 \times 2048$ systems were used and lesser statistics were gathered to obtain similar statistical errors. No finite size effects were noticed. As can be seen from figure 3, (47) with the value $C=1$ taken from [15] 


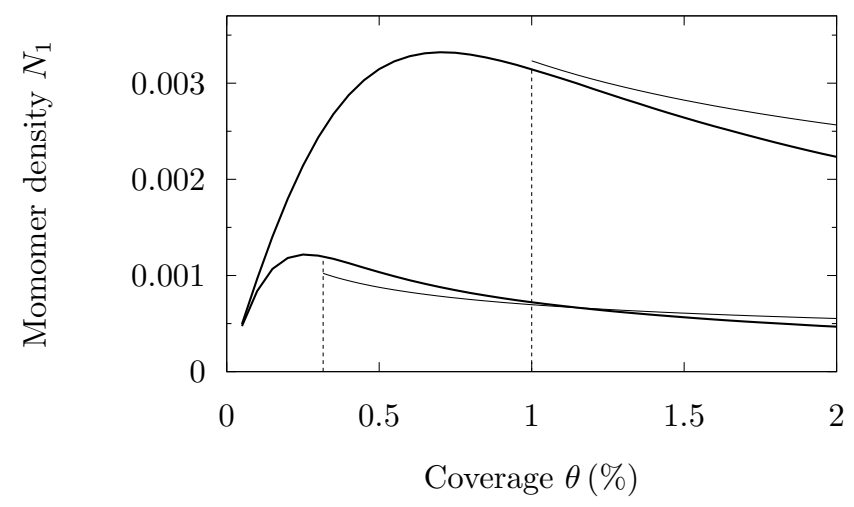

Figure 2. Thick lines: the monomer densities obtained in KMC simulations for two values of the diffusion to deposition rates ratio $R=10^{4}$ (upper line) and $R=10^{5}$ (lower line); thin solid lines: the densities in the steady-state regime calculated according to the asymptotic formula (50); vertical dashed lines show the positions of the crossover coverage $\theta^{*}$ from (49).

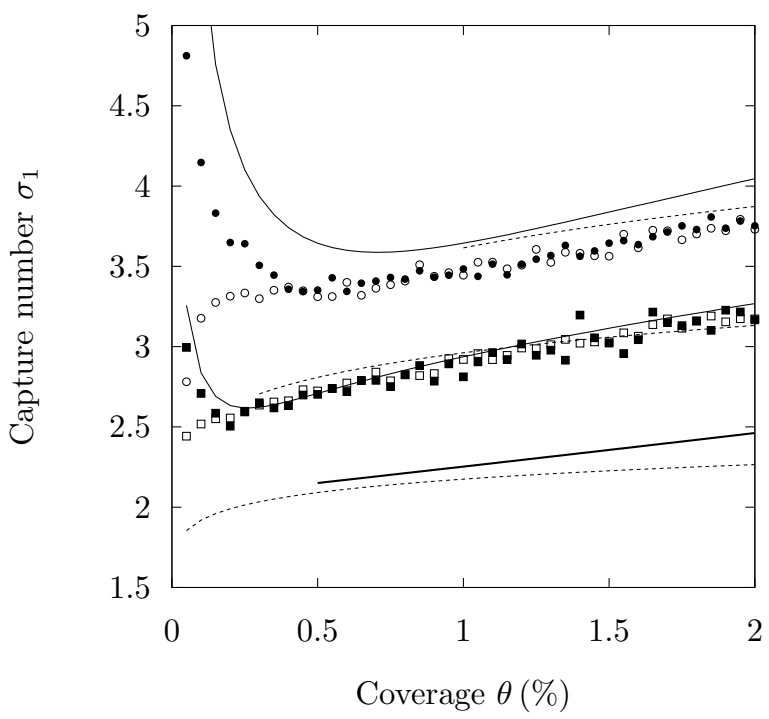

Figure 3. Filled symbols: KMC measurements with the use of the exact formula (23) at diffusion to deposition rates ratios $R=10^{4}(\bullet)$ and $R=10^{5}(\mathbf{\square})$; similar empty symbols: KMC measurements with the use of the procedure described in 20] and [25]. Thin solid lines: $\sigma_{1}$ calculated according to (47) with $C=1$ [15] with the use of the simulated values of $N_{1}$ (see figure 2); dashed lines: (50) for $\theta>\theta^{*}$. Thick solid line: fit to the $R=10^{7} \mathrm{KMC}$ data from figure 2 of [25]. 
semiquantitatively describes the simulated data over the whole range of coverage studied both in the transient regime and in the course of the steady-state growth, especially at the larger diffusion to deposition rates ratio $R$, as predicted theoretically. In the paper [39] similar value of $C \approx 1.38$ was found. Thus, the results of [39, 15] and our results seems to rule out the values $C \sim 32-64$ advocated in [28, 2].

On the other hand, the KMC procedure suggested in [20] and [25] produces results in qualitative disagreement with the exact data in the transient region, though practically coincides with them within the statistical errors in the steady-state regime,as can be seen from figure 3, The reason for this seems to be clear. The procedure in question consists in stopping the deposition from the external source at any given moment and detaching the monomers captured by the islands or separating the nucleated dimer and putting them back into the homogeneous deposition flux over the free sites without occupied neighbours [20, 25]. The capture/nucleation events are counted for every island size. By continuing this arrested growth simulation sufficiently long one can obtain arbitrarily good statistics on capture numbers. Obviously, however, that this procedure may give quite accurate results only in the steady state regime where the diffusion profile $N_{1}(\mathbf{x})$ is time-independent, so the origin of the homogeneously deposited atoms,- - either from the physical deposition flux or from the artificially simulated one,- is irrelevant: the profile is always the same [17].

At small coverages at the early stage of the growth, however, the diffusion profile only strives to reach the steady-state shape and so is time dependent. Thus, at the start of the arrested deposition the diffusion profile has a physical shape which permanently changes with time and as the arrested deposition simulation continues the shape of the profile in the absence of the external deposition became unphysical and the simulation produces incorrect $\sigma_{1}$ values, as can be seen from figure 3 ,

\section{Conclusion}

In this paper we used the second quantization representation of the stochastic master equation governing the coherent epitaxial growth to develop a formalism that allows for a rigorous derivation of the rate equations for the epitaxial growth. The REs obtained in sec. 3 are more detailed than those found in literature in that they describe the evolution of individual island configurations. This may be of value in the cases when the number of configurations is restricted, as in the case of $1 \mathrm{D}$ growth [37, 38, 4]. In the majority of cases, however, the number of the island configurations is too large to be used in practical calculations [35]. Therefore, it is conventional to use the REs where the island densities comprising all island configurations of the same size are used. We illustrated the derivation of such REs in section 4.1. In some cases, however, experimental data show that not all configurations are equal. The so-called magic clusters may play more significant role than islands of other shapes [40, 41]. Our equations of Sec. 3 are capable of assuring a special treatment to such configurations. Furthermore, in the case of quantum dot growth, islands of different morphologies exhibit qualitatively different 
growth behaviours [42, 43] which also would require more detailed characterization of islands than only by their size. Finally, the detailed derivation of the REs can be helpful in better understanding the approximations underlying the REs and the means of improving them with the use of more accurate decouplings.

As we showed in the present paper, even in the simplest case of the irreversible submonolayer growth in precoalescence regime that has been extensively investigated for about forty years there still remain controversial issues that need further investigation. Obviously that in more complex cases such as the currently hot subject of the quantum dot growth [42, 43] the difficulties will enlarge manifold. We expect that the formalism developed in the present paper will prove to be an indispensable tool of derivation of the REs for such cases. The derivation in the framework of the second quantisation formalism, though cumbersome, is quite straightforward. Besides, it is very flexible and do not reduces to the simple case of the hard core bosons we considered in the present paper. Multiple site occupation, coalescence, 3D growth, reversibility, etc all can be accounted for both in the quantum formalism [29, 30, 31, 32, 33] and in the REs [44, 23].

\section{Appendix}

The commutators in (23) can be calculated with the use of the chain rule

$$
\begin{aligned}
{\left[A_{1} A_{2} \ldots A_{k}, X\right]=} & {\left[A_{1}, X\right] A_{2} \ldots A_{k}+A_{1}\left[A_{2}, X\right] A_{3} \ldots A_{k}+\ldots } \\
& +A_{1} \ldots A_{k-1}\left[A_{k}, X\right]
\end{aligned}
$$

the definitions

$$
n_{i}=a_{i}^{+} a_{i}, \quad \bar{n}_{i} \equiv 1-n_{i}=a_{i} a_{i}^{+}
$$

and the commutators

$$
\begin{aligned}
& {\left[n_{i}, a_{i}^{+}\right]=-\left[\bar{n}_{i}, a_{i}^{+}\right]=a_{i}^{+}} \\
& {\left[n_{i}, a_{i}\right]=-\left[\bar{n}_{i}, a_{i}\right]=-a_{i} .}
\end{aligned}
$$

As can be easily seen, in the case of binary site occupancy vector $|\Omega\rangle$ in (19) can be expressed through vectors (3) as

$$
|\Omega\rangle=\prod_{i}\left(|0\rangle_{i}+|1\rangle_{i}\right)
$$

From this representation it is easy to see that inside the matrix elements of type (20), i. e., with vector $|\Omega\rangle$ on the left, the nondiagonal operators can always be turned into diagonal ones as

$$
\begin{aligned}
& \langle\Omega| a_{i}^{+}=\langle\Omega| \bar{n}_{i} \\
& \langle\Omega| a_{i}=\langle\Omega| n_{i} .
\end{aligned}
$$

Care should be taken to keep noncommuting operators in correct order. For example, vital to the results below is that operator $\tilde{a}_{i}$ in (17) is the rightmost in the product. The 
evolution equations that we derive strongly depend on this because of the following not quite intuitive identities:

$$
\begin{aligned}
& \langle\Omega| \bar{n}_{i} a_{i}=\langle\Omega| n_{i} \\
& \langle\Omega| \bar{n}_{i} a_{i}^{+}=0
\end{aligned}
$$

[cf. (A.6) - (A.7)] .

Because physically there exists a qualitative difference between the islands and mobile monomers, it should be reflected in the formalism. Therefore, we will discuss the calculation of the commutators for these entities separately.

\section{Appendix A.1. The island commutators $(s>1)$}

First we calculate the simpler commutator of the deposition term in (24) with the island operator (28). From (A.3) it is seen that the commutator of the creation operators with the island operator (28) will consist of two parts : the commutator with operators $n_{i}$ on interior island sites and with operators $\bar{n}_{i}$ on the border sites. Though the commutators in (A.3) differ only by sign, the results are somewhat different. The border commutators when acting on the vector $\langle\Omega|$ result in the same expression as the island operator (28) itself while the interior commutators lead to islands with one less atom that arise when every $n_{i}$ is sequentially replaced with $\bar{n}_{i}$ corresponding to an empty site. We denote symbolically the set of such islands as $\{c \backslash 1\}$ :

$$
\langle\Omega|\left[\hat{N}_{s, i}^{(c)}, \mathbf{W}^{F}\right]=F\langle\Omega|\left(\sum_{\{c \backslash 1\}} \hat{N}_{s-1, i}^{(c) 1)}-\bar{s} \hat{N}_{s, i}^{(c)}\right),
$$

where the first sum is over $s$ islands whose configurations differ from $c$ by the absence of each of $s$ atoms constituting the initial island. It is to be noted that in some cases this might lead to disconnected sets, i. e., to the appearance of two or more separate islands or monomers. Such cases correspond to the growth with the coalescence and we do not consider them in the present paper devoted to pre-coalescence growth regime. Their inclusion would lead to exact equations of more general type.

The calculation of the commutator with the second term in (24) in the case of islands is very similar due to the structure of $\tilde{a}_{i}$ in (26). Because the annihilation operator in $\mathbf{W}^{D}$ is multiplied by factors $\bar{n}_{i_{\mathrm{NN}}}$ on all neighbour sites, $\tilde{a}_{i}$ will commute with any diagonal operator on site $i$ that contains at least one of $n_{i_{\mathrm{NN}}}$ as a factor. But such are all operators entering $\hat{N}_{s, i}^{(c)}$ by definition. Therefore, in the commutator $\tilde{a}_{i}$ can be treated as a number similar to $F$ and the commutator calculated exactly as in (A.10). The main complication is connected with acting with $\mathrm{D} \sum_{i_{\mathrm{N}}} \tilde{a}_{i_{\mathrm{N}}}$ on the vector $\langle\Omega|$. But as explained above, for every boundary site only the sites that have no neighbours with $n_{i}$ will contribute, that is, only the NN sites to the island with configuration $c$ and the $\mathrm{NN}$ sites to the islands with one less atom [see (A.10)] amounting to

$$
\langle\Omega|\left[\hat{N}_{s, i}^{(c)}, \mathbf{W}^{D}\right]=D \sum_{\{c \backslash 1\}_{\mathrm{NN}}}^{\prime}\langle\Omega| \hat{N}_{1, i_{\mathrm{NN}}} \hat{N}_{s-1, i}^{(c \backslash 1)}-D\langle\Omega| \sum_{i_{\mathrm{NN}}} m_{i_{\mathrm{NN}}} \hat{N}_{1, i_{\mathrm{NN}}} \hat{N}_{s, i}^{(c)},
$$


where the prime over the first sum is to indicate that the summation over the NN sites comprises only those sites (there may be from one to three) which the detached atom can rich in one hop and $m_{i_{\mathrm{NN}}}$ is the number of nucleation paths for the $\mathrm{NN}$ atom to reach the island [23].

\section{Appendix A.2. The monomer case}

Because the monomer is a limiting case of an island, we can proceed with the calculation of commutator as above by only bearing in mind that monomers differ from islands in two respects. First, when an atom is taken away from the monomer in the first terms in (A.10) and (A.11) we obtain an "empty island". In the first case we find

$$
\langle\Omega|\left[\hat{N}_{1, i}, \mathbf{W}^{F}\right]=F\langle\Omega|\left(\hat{N}_{0, i}-4 \hat{N}_{1, i}\right),
$$

where the "empty island" operator

$$
\hat{N}_{0, i}=\bar{n}_{i} \prod_{\hat{e}= \pm \hat{x}, \pm \hat{y}} \bar{n}_{i+\hat{e}}
$$

has formal structure of $\hat{N}_{1, i}$ (29) shown in figure 1 but with the central operator $n_{i}$ replaced with $\bar{n}_{i}$. The second commutator takes the form

$$
\langle\Omega|\left[\hat{N}_{1, i}, \mathbf{W}^{D}\right]=\langle\Omega|\left(-4 D \hat{N}_{1, i}\right)+\langle\Omega| D\left(\sum_{i_{\mathrm{N}}} \hat{N}_{1, i_{\mathrm{N}}} \hat{N}_{0, i}^{\left(i_{\mathrm{N}}\right)}-\sum_{i_{\mathrm{NN}}} m_{i_{\mathrm{NN}}} \hat{N}_{1, i_{\mathrm{NN}}} \hat{N}_{1, i}\right),
$$

where the last two terms in parentheses are analogous to the terms entering (A.11) for the islands while the first term is due to the commutator with $\tilde{a}$ from $W^{D}$ (26) that is absent in the island case. The operator $\hat{N}_{0, i}^{\left(i_{\mathrm{N}}\right)}$ is similar to the empty island operator $\hat{N}_{0, i}$ and can be obtained from it by means of (A.8), namely by replacing one of $\bar{n}_{i_{\mathrm{N}}}$ factors surrounding site $i$ with $n_{i_{\mathrm{N}}}$. Its action is to suppress those configurations in which an atom detaches from an island.

\section{Acknowledgments}

One of the authors (V. I. T.) expresses his gratitude to Université de Strasbourg and IPCMS for their hospitality.

\section{References}

[1] Barabasi A L and Stanley H 1995 Fractal Concepts in Surface Growth. Cambridge University Press, Cambridge

[2] Evans J W, Thiel P A and Bartelt M C 2006 Surf. Sci. Rep. 611

[3] Jansen A 2012 An Introduction to Kinetic Monte Carlo Simulations of Surface Reactions. Lecture Notes in Physics, Vol. 856. Springer, NY

[4] Albao M A, Evans J W and Chuang F 2009 J. Phys. Condens. Matter 21405002

[5] Bartelt M C and Evans J W 1992 Phys. Rev. B 4612675

[6] Zangwill A and Vvedensky D D 2011 Nano Lett. 112092

[7] Tokar V I and Dreyssé H 2008 Phys. Rev. E 77066705 
[8] Tokar V I and Dreyssé H 2009 Phys. Rev. B 80161403

[9] Nandipati G, Shim Y and Amar J G 2010 Phys. Rev. B 81235415

[10] Brune H, Bales G S, Jacobsen J, Boragno C and Kern K 1999 Phys. Rev. B 605991

[11] Ratsch C and Venables J A 2003 J. Vac. Sci. Technol. A 21 S96

[12] Venables J A 1973 Phil. Mag. 27697

[13] Venables J A, Spiller G D T and Hanbucken M 1984 Reports on Progress in Physics 47399

[14] Amar J G, Family F and Lam P M 1994 Phys. Rev. B 508781

[15] Bales G S and Chrzan D C 1994 Phys. Rev. B 506057

[16] Popescu M N, Amar J G and Family F 1998 Phys. Rev. B 64205404

[17] Blackman J A and Mulheran P A 1996 Phys. Rev. B 5411681

[18] Ratsch C, Zangwill A, Šmilauer P and Vvedensky D D 1994 Phys. Rev. Lett. 723194

[19] Mulheran P A and Blackman J A 1996 Phys. Rev. B 5310261

[20] Bartelt M C and Evans J W 1996 Phys. Rev. B 54 R17359

[21] Evans J W and Bartelt M C 2001 Phys. Rev. B 63235408

[22] O'Neill K P, Grinfeld M, Lamb W and Mulheran P A 2012 Phys. Rev. E 85021601

[23] Bales G S and Zangwill A 1997 Phys. Rev. B 55 R1973

[24] Pimpinelli A and Einstein T L 2007 Phys. Rev. Lett. 99226102

[25] Körner M, Einax M and Maass P 2010 Physical Review B 82201401

[26] van Kampen N G 1981 Stochastic Processes in Physics and Chemistry. North-Holland, NY

[27] Li M and Evans J 2003 Surface Science 546127

[28] Li M and Evans J W 2005 Multiscale Model. Simul. 3629

[29] Doi M 1976 J. Phys. A 91465

[30] Siggia E D 1977 Phys. Rev. B 162319

[31] Grassberger P and Scheunert M 1980 Fortschr. Phys. 28547

[32] Mattis D C and Glasser M L 1998 Rev. Mod. Phys. 70979

[33] Tokar V I 1996 Phys. Rev. E 531411

[34] Clarke S and Vvedensky D D 1988 Journal of Applied Physics 632272

[35] Jensen I 2001 J. Stat. Phys. 102865

[36] Myers-Beaghton A K and Vvedensky D D 1990 Phys. Rev. B 425544

[37] Segovia P, Purdie D, Hensenberger M and Baer Y 1999 Nature (London) 402504

[38] Gambardella P, Dallmeyer A, Maiti K, Malagoll M C, Eberhardt W, Kern K and Carbone C 2002 Nature (London) 416301

[39] Tang L H 1993 J. Phys. I France 3935

[40] Voigtländer B, Kästner M and Šmilauer P 1998 Phys. Rev. Lett. 81858

[41] Lai M Y and Wang Y L 1998 Phys. Rev. Lett. 81164

[42] Stangl J, Holý V and Bauer G 2004 Rev. Mod. Phys. 76725

[43] Tokar V I and Dreyssé H 2013 Journal of Physics: Condensed Matter 25045001

[44] Dobbs H T, Vvedensky D D, Zangwill A, Johansson J, Carlsson N and Seifert W 1997 Phys. Rev. Lett. 79897 